\title{
Rastreio de síndrome poliglandular autoimune em uma população de pacientes com diabetes melito tipo 1
}

\author{
Screening for autoimmune polyglandular syndrome in \\ a cohort of patients with type 1 diabetes mellitus
}

Sofia Gouveia', Leonor Gomes', Cristina Ribeiro', Francisco Carrilho'

\begin{abstract}
RESUMO
Objetivo: Caracterizar uma população de pacientes com diabetes melito tipo 1 (DMT1) relativamente à presença de outras entidades autoimunes que permitam estabelecer o diagnóstico de síndrome poliglandular autoimune (SPGA). Sujeitos e métodos: Incluímos 151 pacientes com DMT1. Analisamos os seguintes parâmetros clínicos: gênero, idade atual, duração da doença, antecedentes pessoais de patologia autoimune e antecedentes familiares de diabetes melito. Submetemos cada doente a um estudo laboratorial com o objetivo de detectar a presença de marcadores imunológicos para a tireoidite, insuficiência adrenocortical, gastrite e doença celíaca, e eventual disfunção associada. Resultados: Coorte com $51,7 \%$ homens, idade média atual de $33,4 \pm 13$ anos e duração da doença de $14,4 \pm 9,6$ anos. Antecedentes pessoais de autoimunidade presentes em $2 \%$ da amostra e história familiar de diabetes melito em $31,1 \%$. A frequência de marcadores imunológicos foi de $24 \%$ para a tireoidite, 9,4\% para a insuficiência adrenocortical, 17,2\% para a gastrite e $2 \%$ para a doença celíaca. Foi diagnosticada SPGA em $25,2 \%$ dos pacientes. 0 risco de SPGA e tireoidite autoimune foi superior em mulheres. A duração da doença correlacionou-se diretamente com a presença de autoanticorpos gástricos e inversamente com a positividade dos anticorpos anti-ilhota, antiglutamato descarboxilase e antitirosina fosfatase. Constatou-se a existência de uma associação entre os marcadores imunológi$\cos$ da tireoidite e gastrite, bem como entre a doença celíaca e insuficiência adrenocortical. Conclusão: Atendendo à frequência e ao prognóstico inerente à SPGA, a necessidade de realizar rastreio em pacientes com DMT1 é enfatizada. O diagnóstico atempado de outras doenças autoimunes permitirá individualizar o tratamento e seguimento do doente. Arq Bras Endocrinol Metab. 2013;57(9):733-8
\end{abstract}

Descritores

Poliendocrinopatias autoimunes; diabetes melito tipo 1; doença de Addison; tireoidite; gastrite; doença celíaca

\begin{abstract}
Objective: To characterize a cohort of patients with type 1 diabetes mellitus (T1DM) on the presence of other autoimmune disorders that could establish the diagnosis of autoimmune polyglandular syndrome (APS). Subjects and methods: We included 151 patients with T1DM. The following clinical parameters were analyzed: gender, current age, disease duration, previous history of autoimmune disorders, and familial history for diabetes mellitus. Each patient was analyzed to detect autoimmune markers of thyroiditis, adrenocortical insufficiency, gastritis, and celiac disease, as well as possible associated dysfunctions. Results: A cohort with $51.7 \%$ males, average current age of $33.4 \pm 13$ years and disease duration of $14.4 \pm 9.6$ years was analyzed. Previous history of autoimmunity was found in $2 \%$, and familial history for diabetes mellitus in $31.1 \%$ of the cohort. Frequency of autoimmune markers was $24 \%$ for thyroiditis, $9.4 \%$ for adrenocortical insufficiency, $17.2 \%$ for gastritis, and $2 \%$ for celiac disease. APS was diagnosed on $25.2 \%$ of the patients. APS and autoimmune thyroiditis risk was higher in females. Disease duration correlated directly with gastric autoantibodies, and inversely with positive islet cell, glutamic acid decarboxylase, and tyrosine phosphatase antibodies. We noticed a correlation between autoimmune markers for thyroiditis and gastritis, as well as between celiac disease and adrenocortical insufficiency. Conclusion: Considering APS prevalence and prognosis, the need for APS screening in patients with T1DM is emphasized. Early diagnosis of other autoimmune disorders will enable us to adjust each patient treatment and follow-up. Arq Bras Endocrinol Metab. 2013;57(9):733-8
\end{abstract}

\section{Keywords}

Autoimmune polyendocrinopathies; type 1 diabetes mellitus; Addison disease; thyroiditis; gastritis; celiac disease
Serviço de Endocrinologia, Diabetes e Metabolismo dos Hospitais da Universidade de Coimbra, Centro Hospitalar e Universitário de Coimbra, E.P.E., Portugal
Correspondência para: Sofia Gouveia

Serviço de Endocrinologia, Diabetes e Metabolismo dos Hospitais da Universidade de Coimbra, Centro Hospitalar e Universitário de Coimbra, E.P.E.

Praceta Prof. Mota Pinto 3000-075 - Coimbra, Portugal sofiamgouveia@gmail.com

Recebido em 22/Maio/2013 Aceito em 1/Ago/2013 


\section{INTRODUÇÃO}

A associação de duas patologias autoimunes (sendo pelo menos uma delas do foro endocrinológico) designa-se por síndrome poliglandular autoimune (SPGA). As doenças autoimunes endocrinológicas englobam o diabetes melito tipo l (DMTl), doença de Addison, tireoidite, hipoparatiroidismo, hipofisite linfocítica e hipogonadismo hipergonadotrófico. Podem igualmente estar implicadas patologias do foro não endocrinológico, nomeadamente gastrite autoimune, doença celíaca, doença inflamatória intestinal, hepatite autoimune, cirrose biliar primária, colangite esclerosante, vitiligo, alopecia autoimune, dermatite herpetiforme, síndrome de Sjögren, lúpus eritematoso sistêmico, doença mista do tecido conjuntivo, artrite reumatoide, síndrome antifosfolipídico, anemia hemolítica autoimune, trombocitopenia autoimune, esclerose múltipla, miastenia gravis, stiff-man syndrome (1-6).

As síndromes poliglandulares autoimunes podem subdividir-se em quatro tipos. São classificados como SPGA tipo 1 os pacientes que manifestem pelo menos duas das três manifestações principais (candidíase mucocutânea crônica, hipoparatiroidismo autoimune e doença de Addison autoimune). O diagnóstico de SPGA tipo 2 requer a presença de insuficiência adrenocortical primária autoimune associada à disfunção tireoidiana autoimune e/ou DMTl autoimune (na ausência de hipoparatiroidismo e de candidíase mucocutânea crônica). Por definição, a SPGA tipo 3 refere-se à coexistência de disfunção tireoidiana autoimune e outra patologia autoimune (exceto doença de Addison, hipoparatiroidismo ou candidíase mucocutânea crônica). $\mathrm{O}$ diagnóstico de SPGA tipo 4 implica a associação entre duas entidades autoimunes que não possam ser classificadas como um dos subtipos previamente enunciados (1-8).

Considera-se que uma SPGA é potencial quando (pelo menos) uma das duas patologias indispensáveis ao diagnóstico se encontra em uma fase pré-clínica (com marcadores imunológicos positivos mas ainda sem disfunção confirmada pelo estudo complementar $)(1,3,4)$.

Aproximadamente um em cada três pacientes com DMTl manifesta uma forma de SPGA no momento do diagnóstico ou ao longo da evolução da doença. Em termos cronológicos, a DMTl é frequentemente a primeira manifestação de SPGA (1-3,7-9).

Em um doente com DMTl, as hipoglicemias de repetição aparentemente inexplicáveis podem decorrer do desenvolvimento da doença de Addison, hipotireoidismo ou doença celíaca. Por outro lado, uma tendência injustificada para a hiperglicemia poderá ser o primeiro indício de uma doença de Graves. Em pacientes com DMTl e anemia de novo, deve avaliar-se a hipótese de a anemia ser consequência de déficit de ferro, ácido fólico e/ou vitamina B12 por doença celíaca ou gastrite autoimune. Pela frequência e pelo impacto que as restantes patologias autoimunes podem exercer sobre a qualidade de vida do doente com DMTl, justifica-se a realização de rastreio seriado para a SPGA. O diagnóstico precoce dessa entidade permitirá modificar o plano de seguimento, minimizando a morbimortalidade atribuível primordialmente à insuficiência adrenocortical (1,5-7).

O rastreio imunológico de SPGA em pacientes com DMTl autoimune implica a pesquisa de autoanticorpos para a tireoidite (anticorpos antitireoperoxidase, antitireoglobulina), gastrite autoimune (anticorpos anticélulas parietais, antifator intrínseco), doença celíaca (anticorpos do tipo imunoglobulina A antitransglutaminase tecidual, antiendomísio) e doença de Addison (anticorpos anti-21-hidroxilase, anticélulas do córtex suprarrenal) $(1,3,5-8)$.

Os anticorpos específicos da DMTl incluem os anticorpos anti-ilhota (ICA), antiglutamato descarboxilase 65 (GADA), antitirosina fosfatase IA-2 (IA2A), anti-insulina e antitransportador de zinco (1-8).

Os pacientes com gastrite autoimune podem desenvolver anemia ferropriva, anemia perniciosa (por déficit de vitamina B12), adenocarcinoma gástrico e tumor carcinoide gástrico (pela hipergastrinemia subsequente à acloridria). A doença celíaca pode condicionar anemia por déficit de ferro e/ou de ácido fólico, hipocalcemia, linfoma e carcinoma intestinal $(5,7,8)$.

O objetivo deste trabalho foi avaliar, em uma população de pacientes com DMTl, a presença de outra(s) patologia(s) autoimune(s) (e disfunção associada) que pudesse $(\mathrm{m})$ constituir uma forma de SPGA.

\section{MATERIAIS E MÉTODOS}

O estudo realizado incluiu indivíduos com diabetes melito tipo 1 observados em consulta no nosso serviço no período compreendido entre 1 de julho de 2010 e 31 de março de 2011.

O diagnóstico de DMTl foi considerado confirmado em pacientes que, para além de cumprirem os critérios de diagnóstico de diabetes melito, apresentassem 
anticorpos específicos para a DMTl positivos (anticorpos anti-ilhota, antiglutamato descarboxilase $65 \mathrm{e} / \mathrm{ou}$ antitirosina fosfatase IA-2) e/ou um nível de peptídeo C no momento do diagnóstico inferior a $0,5 \mathrm{ng} / \mathrm{mL}$.

A população selecionada foi caracterizada relativamente a gênero, idade atual, duração da doença, antecedentes pessoais de patologia autoimune e familiares de diabetes melito. Durante o período temporal previamente enunciado, cada elemento da amostra foi submetido a um estudo laboratorial que incluiu, para além dos autoanticorpos específicos da DMTl (anticorpos anti-ilhota, antiglutamato descarboxilase 65 e antitirosina fosfatase IA-2), a pesquisa de marcadores imunológicos de outras doenças, nomeadamente a tireoidite autoimune (anticorpos antitireoperoxidase e antitireoglobulina), doença de Addison autoimune (anticorpos anti-2l-hidroxilase), gastrite autoimune (anticorpos anticélulas parietais e antifator intrínseco) e doença celíaca (anticorpos do tipo imunoglobulina A antitransglutaminase e, quando positivos, anticorpos antiendomísio). Em indivíduos com marcadores imunológicos positivos foi realizada uma avaliação adicional, no sentido de determinar a existência de disfunção associada. Essa avaliação consistiu na determinação da TSH e T4 livre no caso da tireoidite e da ACTH e cortisol das 8 horas da manhã, cortisol após administração de $250 \mu \mathrm{g}$ de tetracosactídeo, ionograma, renina e aldosterona na doença de Addison. Perante gastrite autoimune eram dosadas a gastrina e a vitamina $\mathrm{Bl} 2 \mathrm{e}$, na doença celíaca, o cálcio e o ácido fólico; em ambas as situações eram requisitados um hemograma e o estudo da cinética do ferro. Os pacientes com anticorpos antitransglutaminase positivos eram referenciados à consulta de Gastrenterologia e, posteriormente, submetidos à biópsia do intestino delgado para confirmação do diagnóstico. Não se procedeu ao rastreio de SPGA tipo 1 atendendo ao fato que o doente mais jovem da amostra tinha 13 anos, sendo, portanto, improvável que o diagnóstico dessa entidade não tivesse sido previamente estabelecido nos elementos dessa coorte.

O protocolo elaborado no sentido de pesquisar outras patologias autoimunes resulta das recomendações dirigidas a pacientes com DMTl efetuadas por diferentes autores. $\mathrm{O}$ estudo da disfunção associada baseia-se nas recomendações específicas de cada entidade autoimune $(1,5-8,10,11)$.

Os pacientes incluídos foram alvo de consentimento informado relativo ao estudo. A análise estatística dos dados foi realizada com recurso ao SPSS 18.0. Foi con- siderado como estatisticamente significativo um valor de $p$ inferior a 0,05 .

\section{RESULTADOS}

Foram incluídos 151 pacientes com DMTl, sendo que $51,7 \%$ eram do sexo masculino. A amostra apresentava uma idade média de $33,4 \pm 13$ anos e uma duração média da doença de $14,5 \pm 9,6$ anos. Apenas $2 \%$ da população estudada possuíam antecedentes pessoais de doença autoimune não endocrinológica, correspondendo em $66,7 \%$ dos casos a asma e em $33,3 \%$ a doença mista do tecido conjuntivo. Cerca de $31,1 \%$ dos pacientes possuíam história familiar de diabetes melito: $57,4 \%$ tinham exclusivamente familiares com diabetes melito tipo 2, 27,7\% exclusivamente familiares com diabetes melito tipo 1 e 14,9\% tinham pelo menos um familiar com diabetes melito tipo 1 e outro com diabetes melito tipo 2 .

Os anticorpos específicos da DMTl mais frequentes foram os GADA (doseáveis em 53,8\% da amostra), seguidos pelos IA2A $(30,2 \%)$ e pelos ICA $(30,1 \%)$.

Os anticorpos antitireoperoxidase e/ou antitireoglobulina foram detectados em $24 \%$ da população estudada. Verificou-se que $58,3 \%$ dos pacientes com tireoidite autoimune apresentavam disfunção associada (90,5\% hipotireoidismo e 9,5\% hipertireoidismo).

Os anticorpos anti-21-hidroxilase estavam presentes em $9,4 \%$ da amostra. Contudo, nenhum dos pacientes com autoimunidade positiva para a doença de Addison apresentou alterações laboratoriais compatíveis com o diagnóstico de insuficiência adrenocortical.

Os anticorpos anticélulas parietais e/ou antifator intrínseco foram positivos em $17,2 \%$ dos pacientes estudados. Desse subgrupo, 61,5\% apresentavam hipergastrinemia, $46,2 \%$, deficiência isolada de ferro, $19,2 \%$, deficiência isolada de cobalamina e 3,8\% apresentavam anemia atribuível à deficiência concomitante de ferro e cobalamina.

Os anticorpos antitransglutaminase foram positivos em $2 \%$ da amostra; esses pacientes apresentavam igualmente anticorpos antiendomísio. O diagnóstico de doença celíaca foi confirmado histologicamente por biópsia intestinal. Observou-se deficiência isolada de ferro em $66,7 \%$ desse subgrupo; nenhum desses pacientes evidenciou anemia, deficiência isolada de ácido fólico ou hipocalcemia. Nenhum doente apresentou em simultâneo autoimunidade positiva para gastrite autoimune e doença celíaca. 
Constatou-se que 25,2\% dos pacientes com DMTl reuniam critérios para o diagnóstico de síndrome poliglandular autoimune. Destes, $55,3 \%$ foram classificados como SPGA tipo 3 (DMTl e disfunção tireoidiana autoimune) e $44,7 \%$ como tipo 4 (DMTl e gastrite autoimune ou doença celíaca com disfunção associada). O diagnóstico de síndrome poliglandular autoimune potencial foi estabelecido em $14,6 \%$ da amostra $(45,4 \%$ como tipo $2,36,4 \%$ como tipo 3 e 18,2\% como tipo 4 ).

A frequência de autoanticorpos, disfunção associada a outras entidades autoimunes e de síndrome poliglandular autoimune encontra-se representada nas figuras 1 a 7 .

O risco de SPGA (teste $X^{2} ; p-0,013$; odds ratio- 2,6 ) e de tireoidite autoimune (teste $\mathrm{X}^{2} ; p-0,004 ;$ odds ratio-3,1) foi superior no sexo feminino.

Aplicando a regressão logística (considerando como variáveis independentes a idade atual e a duração da DMTl), verificamos que a duração da doença influencia a presença de marcadores de gastrite autoimune,

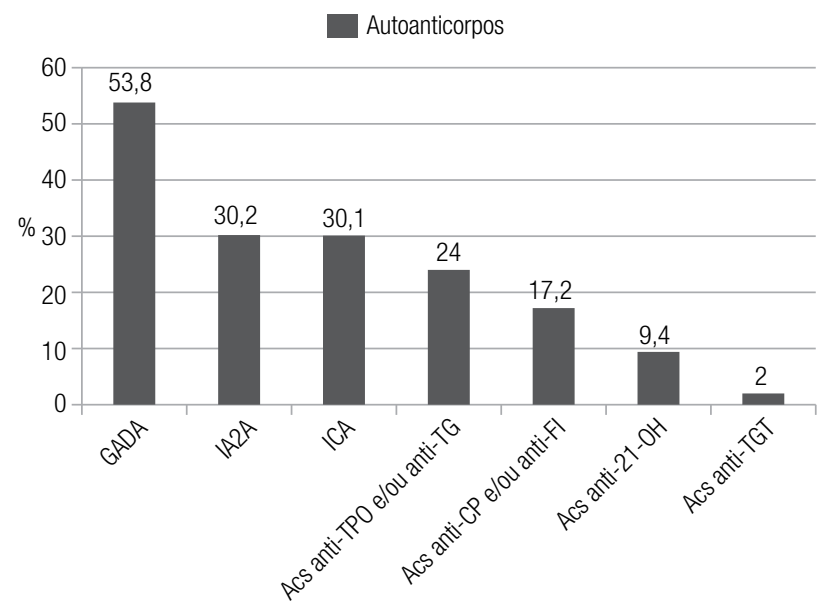

Figura 1. Frequência de autoanticorpos na amostra estudada. GADA: anticorpos antiglutamato descarboxilase 65; IA2A: anticorpos antitirosina fosfatase IA-2; ICA: anticorpos anti-ilhota; Acs: anticorpos; TPO: tireoperoxidase; TG: tireoglobulina; $\mathrm{CP}$ : células parietais; FI: fator intrínseco; 21-OH: 21-hidroxilase; TGT: transglutaminase tecidual.

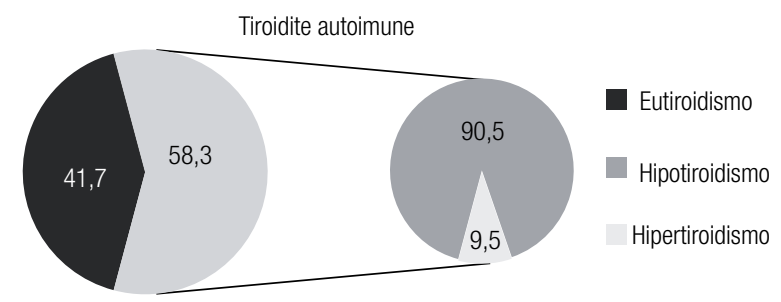

Figura 2. Frequência das manifestações de disfunção em contexto de tiroidite autoimune.
ICA e GADA. Por cada ano que decorre após o diagnóstico de DMTl, a probabilidade de apresentar marcadores de gastrite autoimune aumenta $6,3 \%(p-0,032)$ e de positividade para os ICA e GADA diminui respectivamente $7,7 \%(p-0,004)$ e $7,2 \%(p-0,002)$. Por outro lado, a duração e a idade influenciam, de forma independente, a positividade dos IA2: por cada ano extra de duração e de idade a probabilidade de detecção diminui $9,5 \%(p-0,002)$ e $4,4 \%(p-0,04)$, respectivamente.

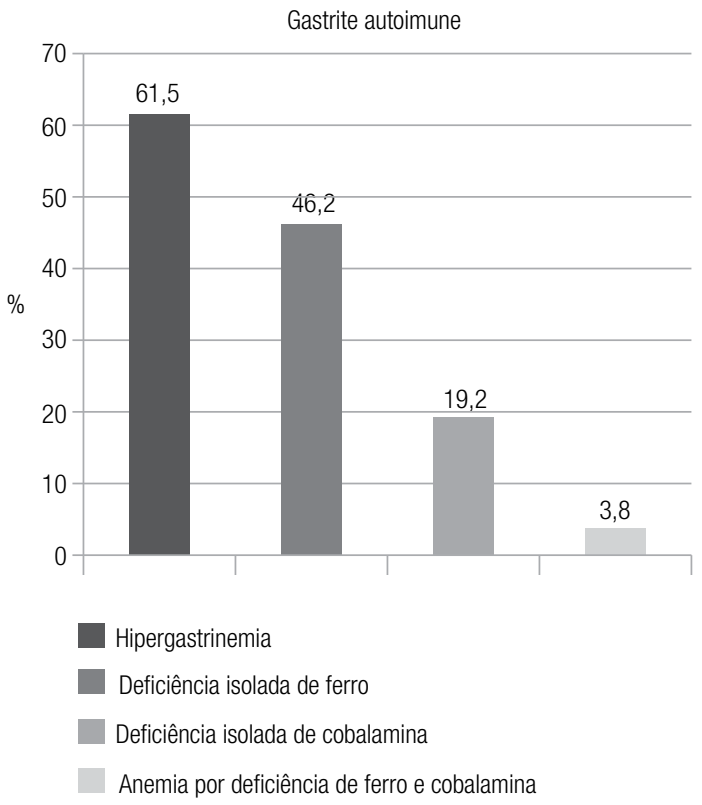

Figura 3. Frequência das manifestações de disfunção em contexto de gastrite autoimune.

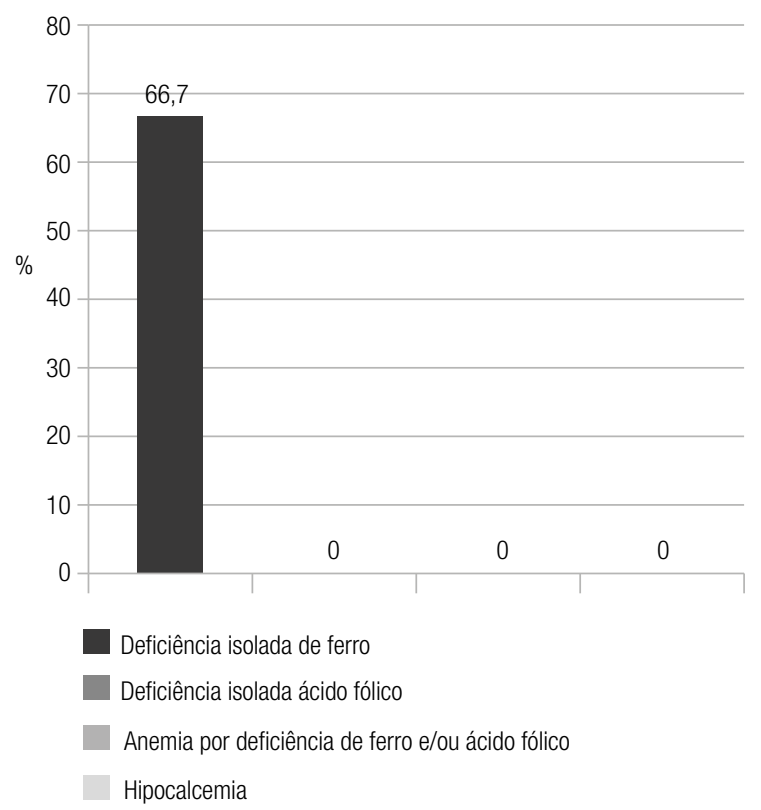

Figura 4. Frequência das manifestações de disfunção em contexto de doença celíaca. 


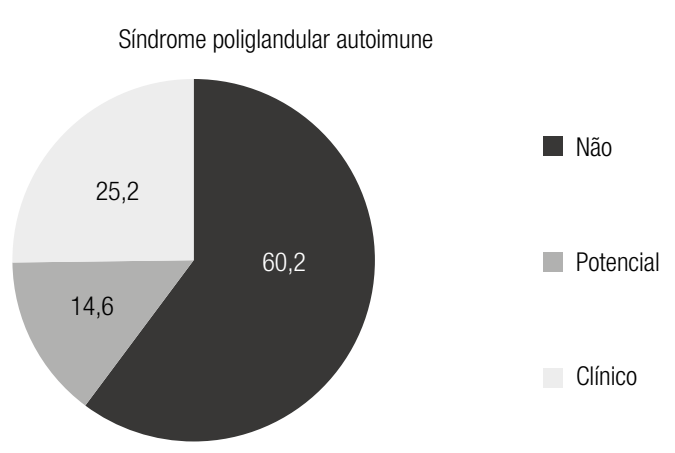

Figura 5. Frequência de síndrome poliglandular autoimune na amostra estudada.

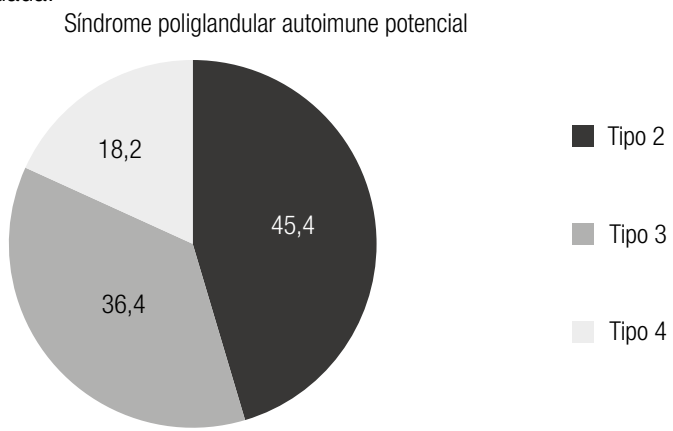

Figura 6. Classificação das síndromes poliglandulares autoimunes potenciais diagnosticadas na amostra estudada.

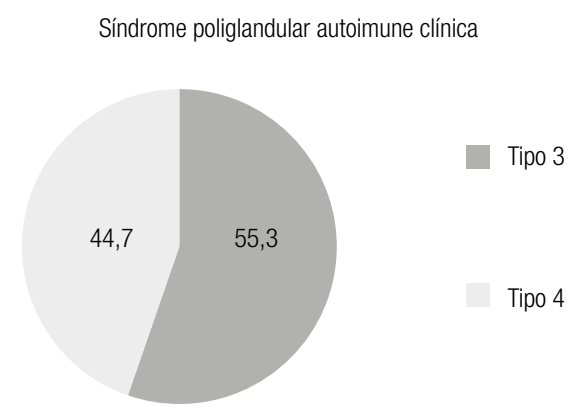

Figura 7. Classificação das síndromes poliglandulares autoimunes clínicas diagnosticadas na amostra estudada.

A presença de marcadores imunológicos de gastrite autoimune associou-se à positividade dos anticorpos antitireoidianos (teste $\mathrm{X}^{2} ; p-0,002 ;$ odds ratio $-3,9$ ). Não foi encontrada associação entre a presença de GADA e anticorpos antitireoidianos e/ou marcadores imunológicos de gastrite autoimune.

Verificou-se também a existência de uma associação entre os anticorpos anti-21-hidroxilase e antitransglutaminase (teste exato de Fisher; $p-0,023$; odds ratio- 22,5 ).

\section{DISCUSSÃO}

A frequência da síndrome poliglandular autoimune nessa população de pacientes com DMTl foi de $25,2 \%$.

As patologias autoimunes mais frequentemente associadas à DMTl foram a tireoidite autoimune (24\%) e a gastrite autoimune $(17,2 \%)$. A tireoidite autoimune, a gastrite autoimune e a doença celíaca associaramse na maioria dos casos à disfunção, estabelecendo o diagnóstico de SPGA. A doença de Addison autoimune constituiu a exceção: nenhum dos pacientes com anticorpos anti-2l-hidroxilase positivos evidenciou alterações analíticas e/ou clínicas que indiciassem insuficiência adrenocortical. Por conseguinte nenhum elemento da amostra foi classificado com o diagnóstico de SPGA tipo 2 clínico. Atendendo à elevada especificidade dos anticorpos anti-21-hidroxilase em pacientes com outras patologias autoimunes previamente diagnosticadas, seu valor preditivo é significativo. A probabilidade de esses pacientes desenvolverem insuficiência adrenocortical é superior caso sejam crianças, do sexo masculino, com título de anticorpos anti-2l-hidroxilase elevado e/ou resultado subnormal na avaliação da função adrenocortical $(2,3,10)$. O título mais elevado de anticorpos anti-21-hidroxilase na nossa amostra foi de $2611 \mathrm{U} /$ $\mathrm{mL}(\mathrm{VR}<\mathrm{l})$, numa jovem de 15 anos. A detecção desse marcador imunológico permitiu-nos identificar os pacientes em risco, alertá-los para os sintomas sugestivos de disfunção e planear seu estudo laboratorial periódico, de modo a detectar e tratar precocemente a insuficiência adrenocortical, melhorando o prognóstico.

Nesta amostra, e à semelhança do que está descrito em outros estudos, o diagnóstico de SPGA e a presença de anticorpos antitireoidianos foram mais frequentes no sexo feminino. O fato de a maioria dos casos de SPGA ser do tipo 3 (com disfunção tireoidiana autoimune associada) poderá justificar a maior prevalência de SPGA em mulheres na população estudada $(5,8,12)$.

Constatou-se na nossa amostra o habitual decaimento dos títulos de anticorpos específicos da DMTl ao longo da evolução da doença (13). Por oposição, a presença de marcadores de gastrite autoimune aumentou com a duração da DMTl, à semelhança do que foi publicado previamente (12).

Obteve-se associação entre marcadores de gastrite autoimune e de tireoidite autoimune na coorte estudada. Está descrito que cerca de $50 \%$ dos pacientes com gastrite autoimune apresentam anticorpos antitireoperoxidase positivos, o que será atribuível à imunidade 
tireogástrica, mais frequente em pacientes com DMTl com persistência dos ICA e/ou dos GADA. O antígeno glutamato descarboxilase está presente em nível do cérebro, pâncreas, tireoide e estômago, o que poderá justificar a associação já documentada entre os GADA e os anticorpos dirigidos contra a tireoide e estômago. No entanto, essa associação com os GADA não foi encontrada na nossa amostra (7).

Observou-se a existência de uma associação entre a positividade dos anticorpos anti-21-hidroxilase e antitransglutaminase. É possível que esses pacientes com DMTl, doença celíaca e anticorpos anti-21-hidroxilase positivos apresentem um haplótipo de risco, nomeadamente $\mathrm{DRBl}{ }^{*} 0301$ (DR3), $\mathrm{DQAl}{ }^{*} 0501$ ou DQB1 *0201 (DQ2), DQAl*0301 ou DQB1*0302 (DQ8) ou um polimorfismo no alelo 5.1 do gene MIC-A (MHC I-related gene A). Contudo, essa hipótese não foi investigada $(8,14)$.

O rastreio de SPGA nesta população de pacientes com DMTl permitiu-nos concluir que $25,2 \%$ dos elementos da amostra apresentavam SPGA e 14,6\%, SPGA potencial. Trata-se de um número muito significativo de pacientes que se beneficiarão de um plano de seguimento individualizado e da instituição de medidas terapêuticas adequadas, com repercussão vantajosa sobre sua qualidade de vida. As síndromes poliglandulares autoimunes mais frequentes foram a tipo 3 e 4 , o que está de acordo com as recomendações clínicas para o rastreio de algumas componentes específicas da SPGA em indivíduos com DMTl $(15,16)$. Os resultados obtidos e os benefícios que antecipamos que resultem do diagnóstico precoce de patologia autoimune fundamentam a necessidade de um rastreio mais completo de SPGA em pacientes com DMTl.

Declaração: os autores declaram não haver conflitos de interesse científico neste estudo.

\section{REFERÊNCIAS}

1. Owen CJ, Cheetham TD. Diagnosis and management of polyendocrinopathy syndromes. Endocrinol Metab Clin N Am. 2009;38:419-36.
2. Falorni A, Laureti S, Santeusanio F. Autoantibodies in autoimmune polyendocrine syndrome type II. Endocrinol Metabol Clin N Am. 2002; 31:369-89.

3. Betterle C, Dal Pra C, Mantero F, Zanchetta R. Autoimmune adrenal insufficiency and autoimmune polyendocrine syndromes: autoantibodies, autoantigens, and their applicability in diagnosis and disease prediction. Endocrine Reviews. 2002;23:327-64.

4. Queiroz MS. Diabetes melito tipo 1 no contexto das poliendocrinopatias auto-imunes. Arq Bras Endocrinol Metab. 2008;52:198-204.

5. Schatz DA, Winter WE. Autoimmune polyglandular syndrome II: clinical syndrome and treatment. Endocrinol Metabol Clin N Am. 2002;31:339-52.

6. Michels AW, Eisenbarth GS. Autoimmune polyendocrine syndrome type 1 (APS-1) as a model for understanding autoimmune polyendocrine syndrome type 2 (APS-2). J Intern Med. 2009;265:530-40.

7. Van den Driessche A, Eenkhoorn V, Van Gaal L, De Block C. Type 1 diabetes and autoimmune polyglandular syndrome: a clinical review. Neth J Med. 2009;67:376-87.

8. Barker JM. Clinical review: type 1 diabetes-associated autoimmunity: natural history, genetic associations, and screening. J Clin Endocrinol Metab. 2006;91(4):1210-7.

9. Triolo TM, Armstrong TK, McFann K, Yu L, Rewers MJ, Klingensmith GJ, et al. Additional autoimmune disease found in $33 \%$ of patients at type 1 diabetes onset. Diabetes Care. 2011;34(5):1211-3.

10. Coco G, Dal Pra C, Presotto F, Albergoni MP, Canova C, Pedini B, et al. Estimated risk for developing autoimmune Addison's disease in patients with adrenal cortex autoantibodies. J Clin Endocrinol Metab. 2006;91(5):1637-45.

11. van der Windt DAWM, Jellema P, Mulder CJ, Kneepkens CMF, van der Horst HE. Diagnostic testing for celiac disease among patients with abdominal symptoms: a systematic review. JAMA. 2010;303:1738-46.

12. Warncke K, Fröhlich-Reiterer EE, Thon A, Hofer SE, Wiemann D, Holl RW; DPV Initiative of the German Working Group for Pediatric Diabetology; German BMBF Competence Network for Diabetes Mellitus. Polyendocrinopathy in children, adolescents, and young adults with type 1 diabetes: a multicenter analysis of 28,671 patients from the German/Austrian DPV-Wiss database. Diabetes Care. 2010;33(9):2010-2.

13. Masharani U, German MS. Pancreatic Hormones and Diabetes Mellitus. In: Gardner DG, Shoback D. Greenspan's Basic \& Clinical Endocrinology. 9th edition. McGraw-Hill Companies; 2011. p. 573-655.

14. Robles DT, Fain PR, Gottlieb PA, Eisenbarth GS. The genetics of autoimmune polyendocrine syndrome type II. Endocrinol Metabol Clin N Am. 2002;31:353-68.

15. American Diabetes Association. Standards of medical care in diabetes--2013. Diabetes Care. 2013;36 Suppl 1:S11-66.

16. Global IDF/ISPAD Guideline for Diabetes in Childhood and Adolescence. 2011:123-8. Disponível em: http://www.ispad. org/resource-type/idfispad-2011-global-guideline-diabeteschildhood-and-adolescence. Acesso em: 16 fev, 2013. 